\title{
Sur la conjonction du littéraire, du religieux et du savoir existentiel dans les Mémoires d'outre-tombe de Chateaubriand
}

\author{
About the conjunction between literature, religion \\ and existential knowledge in the \\ "Memoirs from Beyond the Grave" of Chateaubriand
}

\author{
Aleksandra Kamińska \\ Université de Szczecin
}

\begin{abstract}
The aim of this paper is to present the relationship between literature, religion and existential knowledge in the memorial work of François-René de Chateaubriand. The first main problem is the transmission of universal values represented by Christianity. The second problem are narrator's tragic experiences that become a source of precious knowledge communicated to the reader. These narrative stakes, reinforced by rhetorical persuasion, are crucial to ensure immortality of the author through the pertinence of his voice addressed to posterity.
\end{abstract}

Keywords: literature, existential knowledge, religion, connivance with the reader, rhetorical persuasion, belief in literary immortality

\section{INTRODUCTION}

La lecture d'un texte littéraire ne se limite pas à la compréhension mais, en tant que lieu de rencontre entre écrivain et son public, s'exerce en fonction de la sensibilité à la problématique exposée. De même, l'activité de l'écrivain ne se restreint pas à la rédaction en évoluant, par ses qualités d'observation, vers une nouvelle perspective d'appréhender la réalité. Ainsi, le texte littéraire est ancré au cœur de l'engagement et de l'interaction, entités qui conditionnent le caractère des connaissances insérées dans toute œuvre littéraire. Ces connaissances ne sont jamais gratuites d'autant que le texte littéraire, plus que tout autre type de production, vise un effet concret et s'inscrit dans une stratégie plus complexe. En ce qui concerne l'écrivain, cette stratégie se résume dans l'efficacité recherchée qui est en mesure de solliciter l'imagination interprétative et figurative du 
lecteur. Celui-ci, de son côté, considère l'œuvre littéraire comme un enrichissement de sa culture personnelle qui s'accomplit par la confrontation à de nouveaux horizons de perception. Ainsi s'instaure un lien imaginaire entre écrivain et lecteur permettant de renouer le nœud de réflexion capable de transformer des expériences personnelles en une quête d'un sens plus profond. L'exploitation du vécu personnel comme un moyen d'action susceptible d'influencer le lecteur et de provoquer sa réflexion semble s'intensifier dans le genre autobiographique et celui de mémoires. C'est là un réseau d'interdépendances qui nous encourage à redéfinir la fonction et l'utilité des connaissances auxquelles se heurte le lecteur dans cette œuvre monumentale de Chateaubriand que constituent les Mémoires d'outre-tombe.

\section{LE MEMORIALISTE ET LES TRANSFORMATIONS SOCIALES}

Le narrateur chateaubrianesque assiste à la monumentalité d'événements historiques imposés par la Révolution, l'Empire et la Révolution de Juillet. Le cataclysme social et toutes ses horreurs se reflètent dans la brutalité des images qui pullulent dans ses Mémoires. Ainsi, les contemporains de Chateaubriand transformés en fossoyeurs de la civilisation forcent l'individu de quitter le giron familial plein de tendresse et d'affection. La prise de la plume se montre poignante, car les impressions du narrateur frappent par une constance troublante des mêmes thèmes et réflexions. Cependant, la décomposition de son univers et la perte de ses repères identitaires en tant que fils, frère, ami et sujet fidèle du roi ne s'opèrent pas à un niveau individuel et local; la tragédie personnelle devient un terreau de considérations sur l'existence humaine et le destin commun. En conséquence, la relation des épreuves personnelles s'érige en transmission du savoir général d'ordre existentiel, social, politique, historique, mais également littéraire et religieux. L'anéantissement de la culture chrétienne visé par les bestialités révolutionnaires incite l'écrivain à repenser la place et le rôle de la religion dans la nouvelle société. La violence immonde et la désacralisation du vieil ordre doivent également donner lieu à des méditations sur l'avenir de la littérature. Chateaubriand est conscient de ce que la puissance de la représentation littéraire réside non seulement dans le récit des atrocités vécues, mais avant tout dans l'opposition et la capacité de manifester ses réactions face aux perversions inouïes. Le narrateur se voit moralement obligé de guider le lecteur vers la vérité qui doit résonner plus fort que la dépravation et le mépris pour le savoir, la religion et la littérature, trois grands piliers de la civilisation européenne. Dans la perspective chateaubrianesque le savoir, la religion et la littérature accèdent au rang des valeurs qui construisent sa vision du monde et qu'il veut communiquer à son public. Puisque la communion entre écrivain et lecteur suppose la croyance et le recours à la pratique interrelationnelle, nous nous 
proposons d'étudier la façon dont le narrateur grave ces trois emblèmes de la civilisation dans la conscience du lecteur. Ces interrogations se montrent d'autant plus pertinentes que la société actuelle est sillonnée par la différenciation et le pluralisme. Il s'agit donc de s'interroger sur l'efficacité des procédés déployés par l'écrivain dans le but de captiver l'attention et l'imagination du lecteur. Le rôle que Chateaubriand accorde au savoir, à la religion et à la littérature exigerait d'ailleurs quelques éclaircissements vu la célérité des transformations évoquées dans l'œuvre. Leur progression interminable met en péril la stabilité de tout artefact humain, y compris l'intelligence des Mémoires auprès des générations suivantes. Il est vrai que d'autres chercheurs, comme Agnès Verlet, ont déjà insisté sur le topos de la vanité chez Chateaubriand mémorialiste (Verlet, 2001). Cependant, on note l'absence d'études portant sur le rôle des moyens rhétoriques qui permettent au narrateur de nuancer son statut à l'intérieur de l'œuvre.

\section{LE MEMORIALISTE ET LA LITTERATURE}

Il est impossible d'aborder le statut du savoir et de la religion sans prendre en considération les fonctions esthétiques que l'écrivain confère à son œuvre. $\mathrm{Vu}$ l'énormité de dépossessions auxquelles se heurte Chateaubriand, les Mémoires surgissent comme un défi de se mesurer avec la réalité hostile et les agissements du Temps. Effectivement, son œuvre est le témoignage significatif d'une relation complexe qui s'établit entre l'écrivain et son époque. Le caractère inévitable des transformations sociales et la sensibilité excessive face à la fugacité de l'existence humaine font naître des ambitions posthumes. L'écrivain est préoccupé d'ériger un monument littéraire grâce auquel il parviendrait à fixer son nom et sa gloire dans la conscience de la postérité. En même temps, la brutalité des renversements ironiques observables dans sa contemporaineté entraîne des doutes sur la survie de l'œuvre littéraire et le principe de l'immuabilité de l'art. Ainsi, l'écrivain se retrouve dans une impasse : s'il ose rappeler au lecteur la fugacité des œuvres littéraires et de toute renommée, il réclame implicitement la nullité de son propre héritage littéraire :

On retrouvait partout Milton et Shakespeare. Montmorency, Biron, Sully, tour à tour ambassadeurs de France auprès d'Élisabeth et de Jacques $\mathrm{I}^{\mathrm{er}}$, entendirent-ils jamais parler d'un baladin, acteur dans ses propres farces et dans celles des autres ? Prononcèrent-ils jamais le nom, si barbare en français, de Shakespeare ? Soupçonnèrent-ils qu'il y eût là une gloire devant laquelle leurs honneurs, leurs pompes, leurs rangs viendraient s'abîmer ? (MDT, 2004 : I, 501) ${ }^{1}$.

${ }^{1}$ Liste des sigles utilisés : MDT - Mémoires d'outre-tombe avec le numéro du volume en chiffres romains, suivi du numéro de la page en chiffres arabes. Cette abréviation désigne notre édition de référence, établie et annotée par Jean-Claude Berchet (2004). 
D'une part, il n'est pas surprenant de constater que les réticences deviennent une arme précieuse par laquelle l'instance narrative tente d'occulter la problématique de la désactualisation de l'art dans son œuvre. De l'autre, en reconnaissant quelques imperfections de la littérature, telle l'indignité de la thématique, le narrateur délégue toute la responsabilité sur la médiocrité politique et morale de ses contemporains :

En dernier résultat $\mathrm{M}$. Thiers, pour sauver son système, a réduit la France à un espace de quinze lieues qu'il a fait hérisser de forteresses ; nous verrons bien si l'Europe a raison de rire de cet enfantillage du grand penseur.

Et voilà comment, entraîné par ma plume, j'ai consacré plus de pages à un homme incertain d'avenir que je n'en ai donné à des personnages dont la mémoire est assurée ? C'est un malheur du trop long vivre : je suis arrivé à une époque de stérilité où la France ne voit plus courir que des générations maigres [...]. Ces Mémoires diminuent d'intérêt avec les jours survenus, diminuent de ce qu'ils pouvaient emprunter de la grandeur des événements $[\ldots]$.

Vous étiez plus heureux, Thucydide et Plutarque, Salluste et Tacite, quand vous racontiez les partis qui divisaient Athènes et Rome! vous étiez certains du moins de les animer, non seulement par votre génie, mais encore par l'éclat de la langue grecque et la gravité de la langue latine ! Que pourrions-nous raconter de notre société finissante, nous autres Welches, dans notre jargon confiné à d'étroites et barbares limites ? Si ces dernières pages reproduisaient nos rebâchages de tribune, ces éternelles définitions de nos droits, nos pugilats de portrefeuilles, seraient-elles, dans cinquante ans d'ici, autre chose que les inintelligibles colonnes d'une vieille gazette ? Sur mille et une conjectures, une seule se-trouverait-elle vraie ? (MDT, 2004 : II, 955).

L'empressement à sauvegarder la légitimité de son œuvre incite le narrateur à amplifier le recours à la satire dont le rire est la transcription littéraire d'une situation insupportable. Même si cet aménagement d'ordre persuasif minimise l'abaissement ironique de sa production littéraire, le rôle de l'écrivain se voit diminué au rang de celui d'un observateur de son siècle qui s'affuble du masque d'un chroniqueur. Vu l'inefficacité de la raillerie excessive, nous n'avons pas l'intention de nous pencher sur la satire qui, en tant que sociocritique, a été d'ailleurs soigneusement étudiée par Ferdinand Ferrys Moukete (Moukete, 2012). De même, lorsque le narrateur médite sur l'abscence de réciprocité entre l'écrivain et son public, l'obsolescence de la thématique et l'inutilité de l'effort scriptural face à l'indifférence des lecteurs, il refuse d'assumer pleinement la responsabilité d'un échec éventuel :

nous autres auteurs, petits prodiges d'une ère prodigieuse, nous avons la prétention d'entretenir des intelligences avec les races futures ; mais nous ignorons, que je crois, la demeure de la postérité, nous mettons mal son adresse. Quand nous nous engourdirons dans la tombe, la mort glacera si dur nos paroles, écrites ou chantées, qu'elle ne se fondront pas comme les paroles gelées de Rabelais (MDT, 2004 : I, 537). 
En l'occurence, le déclin de la responsabilité se manifeste dans l'emploi du pronom personnel « nous » qui permet d'atténuer la faillibilité de l'écrivain et d'étendre la responsabilité à l'ensemble de ses confrères. Toutefois, la référence aux ruses persuasives n'est pas capable de remédier à la dégradation de la position dominante du narrateur dans l'œuvre. La spécificité de la littérature réside dans l'engagement, la prise de position et la capacité de transmettre sa vision au lecteur. L'intention de réfuter l'idée de sa propre insignifiance suppose donc la tâche d'accroître sa crédibilité aux yeux du public, crédibilité par laquelle l'écrivain peut récupérer la fermeté et la fiabilité de son discours. Vu ses aspirations à la grandeur et à l'immortalité, il est primordial qu'il fonde son œuvre sur la monumentalité de la thématique et la noblesse des idées qu'il prétend communiquer au lecteur. Il semble que la démonstration de son pouvoir et de son influence peut s'accomplir par l'enracinement de sa production littéraraire dans l'universalité de la perspective humaniste :

Quand ces mémoires posthumes paraîtront, la polémique quotidienne, les événements pour lesquels on se passionne à l'heure actuelle de ma vie, les adversaires que je combats, même l'acte du bannissement de Charles X et de sa famille, compteront-ils pour quelque chose ? c'est là l'inconvénient de tout journal : on y trouve des discussions animées sur des sujets devenus indifférents; le lecteur voit passer comme des ombres une foule de personnages dont il ne retient pas même le nom : figurants muets qui remplissent le fond de la scène. Toutefois c'est dans ces parties arides des chroniques que l'on recueille les observations et les faits de l'histoire de l'homme et des hommes (MDT, 2004 : II, 517).

Dans les Mémoires, l'abattement du narrateur par la violence et la cruauté d'événements de l'époque produit la réflexion sur la condition humaine infatigable dans la recherche de la vérité et de la légitimité de son chemin. Ainsi, le narrateur chateaubrianesque tire-t-il parti de ses expériences pénibles et s'assure l'intérêt du public en l'emmenant dans la quête de spiritualité et de connaissances véridiques qui préoccupe l'être humain depuis sa création.

\section{LE MEMORIALISTE ET LA RELIGION}

Nous voyons que les bouleversements de l'époque placent le narrateur des Mémoires dans l'incapacité d'éviter tout engagement : quelle que soit sa nature, Chateaubriand est accablé par l'imminence des choix politiques, sociaux et surtout moraux. Il est donc temps d'examiner quels rapports sont entretenus par l'écrivain avec la religion, vu les monstruosités de la révolution, la progression de l'athéisme et du subjectivisme éthique. Cette thématique mérite un traitement exclusif non parce que le christianisme demeure la religion de l'enfance, mais 
parce que, malgré la dévastation de son univers, le narrateur manifeste une remarquable fidélité à ses racines chrétiennes. La scène de la première communion illustre à merveille que le narrateur considère la religion comme la source de son identité humaine qui se traduit par l'adhésion explicite aux obligations de la morale :

J'ose dire que c'est de ce jour que j'ai été créé honnête homme; je sentis que je ne survivrais jamais à un remords : quel doit donc être celui du crime, si j'ai pu tant souffrir pour avoir tu les faiblesses d'un enfant! Mais combien elle est divine cette religion qui se peut emparer ainsi de nos bonnes facultés! Quels préceptes de morale suppléeront jamais à ces institutions chrétiennes ? (MDT, 2004 : I, 180).

$\mathrm{Vu}$ la violence de la désacralisation qui s'attaque au vieil ordre légitime, il n'est pas surprenant que l'écrivain transforme l'acte scriptural en un témoignage susceptible d'éclairer le lecteur sur l'immensité de destruction, d'irrévérence et de profanation :

A droite et à gauche du chemin, se montraient des châteaux abattus ; de leurs futaies rasées, il ne restait que quelques troncs équarris, sur lesquels jouaient les enfants. On voyait des murs d'enclos ébréchés, des églises abandonnées, dont les morts avaient été chassés, des clochers sans cloches, des cimetières sans croix, des saints sans tête et lapidés dans leurs niches (MDT, 2004 : I, 614).

La démesure et l'irrespect poussent le narrateur à remanier le récit en sorte qu'il puisse rendre compte de la totalité de crimes révolutionnaires. Cette tâche narrative se réalise dans notre cas par le ralentissement de l'expression capable d'attirer l'attention du lecteur. La présence de la conjonction de coordination « et» répartit l'expression en deux tableaux en soulignant l'intensité de l'affliction: " des saints sans tête et lapidés dans leurs niches ». Cependant, l'activité du mémorialiste ne se limite pas à la déformation du langage par laquelle le narrateur assaille le lecteur et mime son déchirement intérieur. Au contraire, la turbulence des événements révolutionnaires devient un prétexte pour les considérations générales sur l'homme et sa quête de spiritualité par laquelle il impose au monde sa supériorité. Ainsi, le narrateur chateaubrianesque ne cesse de démontrer que les croyances religieuses croissent même sur les ruines de la civilisation, parce que l'être humain, dans le cours de son histoire, ressent un besoin intarissable de foi et de surnaturel :

Ce fut au milieu des débris de nos temples que je publiai le Génie du Christianisme. Les fidèles se crurent sauvés : on avait alors un besoin de foi, une avidité de consolations religieuses, qui venaient de la privation de ces consolations depuis de longues années. Que de forces surnaturelles à demander pour tant d'adversités subies ! Combien de familles mutilées avaient à chercher auprès du Père des hommes les enfants qu'elles avaient perdus ! Combien de cœurs brisés, combien d'âmes devenus solitaires, appelaient 
une main divine pour les guérir ! On se précipitait dans la maison de Dieu, comme on entre dans la maison du médecin le jour d'une contagion. Les victimes de nos troubles (et que de sortes de victimes !) se sauvaient à l'autel ; naufragés s'attachant au rocher sur lequel ils cherchaient leur salut (MDT, 2004 : I, 640).

La déception causée par le pragmatisme, le rationalisme et le progrès prouvent la spécificité de la condition humaine qui, enfermée dans son dualisme, se voit constamment dominée par l'obsession du réconfort spirituel dans lequel l'homme pourrait retrouver le sens de son existence. Ainsi, la pensée chateau brianesque s'adapte aux dilemmes séculaires qui ont préoccupé l'humanité dans le passé et qui l'absorbent dans la modernité, où l'éloignement de l'Église ne se résout pas nécessairement dans l'athéisme, mais progresse vers la dangérosité de mouvements sectaires. Quoqu'il en soit, Chateaubriand est conscient de ce que la croyance s'appuie sur l'efficacité de la séduction par laquelle l'instance narrative acquiert le pouvoir d'amener le lecteur au renouvellement de la foi. Par conséquent, il soumet son discours aux règles de l'écriture engagée qui, par le déploiement de preuves persuasives, a pour objectif de rediriger le lecteur vers la beauté, la noblesse et l'intelligence de la religion chrétienne. Nous assistons donc à la consécration de la thématique chrétienne qui se récapitule dans la concordance entre la grandeur de l'idée et la noblesse de la représentation ${ }^{2}$. Premièrement, ce discours élevé et pathétique est capable de rassembler le public et de créer une communauté imaginaire par le recours à l'amplification et ses figures majeures telles que la répétition, l'énumération, la gradation et la comparaison. Deuxièmement, le narrateur se rend compte de ce que la fiabilité s'obient aussi par la clarté. Par conséquent, il se tourne vers la simplicité de l'épithète et de la périphrase permettant de juxtaposer la souffrance inhérente à l'existence humaine avec la puissance consolatrice de la foi chrétienne. L'idée de la prééminence de la religion chrétienne dans les Mémoires n'est autre chose que la réconciliation entre des éléments antithétiques éparpillés dans le texte. Remarquons que le topos de la disparition représenté par les épithètes: " adversités subies », " familles mutilées », " cœurs brisés », "âmes devenus solitaires » s'oppose aux périphrases précieuses et ennoblissantes : " force surnaturelle », " main divine », " Père des hommes », " maison de Dieu ». Le nivellement des tensions relève du défi purement persuasif où la place centrale est accordée à la métaphore. Son utilité rhétorique réside dans la capacité d'intégrer le divers, où les transferts métonymiques de la cause à l'effet annihilent le sentiment de douleur et de persécution en faveur de la consolation apportée par la majesté de la religion chrétienne. Comme nous le voyons, le narrateur chateaubrianesque se livre à l'exercice de l'éloquence sacrée, même si

\footnotetext{
${ }^{2}$ Selon Quintilien, l'appropriation de la dispositio et de l'elocutio garantit à l'orateur la communion des sentiments avec le public. Sur ces questions, voir Quintilien (1989: 61).
} 
ce nouveau prédicateur s'éloigne de la monotonie en dotant son discours de la mouvance romantique :

La tendresse filiale que je conservais pour madame de Chateaubriand était profonde. Mon enfance et ma jeunesse se liaient intimement au souvenir de ma mère ; tout ce que je savais me venait d'elle. L'idée d'avoir empoisonné les vieux jours de la femme qui me porta dans ses entrailles, me désespéra : je jetai au feu avec horreur des exemplaires de l'Essai, comme l'instrument de mon crime ; s'il m'eût été possible d'anéantir l'ouvrage, je l'aurais fait sans hésiter. Je ne me remis de ce trouble que lorsque la pensée m'arriva d'expier mon premier ouvrage par un ouvrage religieux : telles fut l'origine du Génie du Christianisme.

"Ma mère", ai-je dit dans la première préface de cet ouvrage, "après avoir été jetée à soixante-douze ans dans des cachots, où elle vit périr une partie de ses enfants, expira enfin sur un grabat, où ses malheurs l'avaient reléguée. Le souvenir de mes égarements répandit sur ses derniers jours une grande amertume ; elle chargea, en mourant, une de mes sœurs de me rappeler à cette religion dans laquelle j'avais été élevé. Ma sœur me manda le dernier vœu de ma mère. Quand la lettre me parvint au-delà des mers, ma sœur elle-même n'existait plus ; elle était morte aussi des suites de son emprisonnement. Ces deux voix sorties du tombeau, cette mort qui servait d'interprète à la mort, m'ont frappé. Je suis devenu chrétien. Je n'ai point cédé, j'en conviens, à de grandes lumières surnaturelles : ma conviction est sortie du cœur; j'ai pleuré et j'ai cru” (MDT, 2004 : I, 553-554).

Il est évident que le narrateur n'a pas l'intention de réduire la portée de l'insinuation rhétorique qui progresse par le ralentissement des métaphores, des épithètes et des périphrases. Au contraire, le freinage rhétorique s'allie à la saturation sémantique en assurant la promotion des idées préromantiques qui rompent avec la rationalité et le bon sens. L'écrivain est dorénavant représenté comme un être supérieur qui reçoit le souffle surnaturel, s'élève vers la transcendance métaphysique et s'expose au conflit inéluctable avec son entourage incapable de percer les mystères du génie. Le moi chateaubrianesque assume ainsi la pluralité romantique de son être: la solitude résultant de l'incompréhension par ses contemporains se compense dans le dévouement à la religion et le passage à l'acte scriptural. Prédisposé à la transcendance, le narrateur ne peut manquer de déployer devant le lecteur la vision tragique de son existence qu'il extériorise par la dramatisation des circonstances pathétiques dans lesquelles naît sa veine littéraire. L'exagération de l'importance de la cause est l'invention majeure de rerum magnitudo, convention littéraire dont se sert le narrateur afin de promouvoir ses déchirements, son exaltation et ses luttes internes. Cette connexion entre religion et littérature que revendique Chateaubriand n'est pas fortuite et révèle à grand fracas sa crainte : effrayé par l'enlisement de ses Mémoires dans l'oubli, à l'instar de l'héritage littéraire de ses éminents prédécesseurs, il place son œuvre monumentale sous les auspices de l'ordre 
durable et légitime que constitue la religion chrétienne. Ainsi, Chateaubriand ne se résigne pas au déclin de la religion, mais s'évertue à ranimer la foi chrétienne par le recours à des formes de spiritualité marginales liées au peuple et à sa ferveur religieuse :

Pie VII cheminait au milieu des cantiques et des larmes, au son des cloches, aux cris de : "Vive le pape ! Vive le chef de l'Eglise !". On lui apportait, non les clefs des villes, des capitulations trempées de sang et obtenues par le meurtre, mais on lui présentait des malades à guérir, de nouveaux époux à bénir au bord de sa voiture ; il disait aux premiers : "Dieu vous console !". Il étendait sur les seconds ses mains pacifiques; il touchait de petits enfants dans les bras de leurs mères. Il ne restait aux villes que ceux qui ne pouvaient marcher. Les pèlerins passaient la nuit sur les champs pour attendre l'arrivée d'un vieux prêtre délivré. Les paysans, dans leur naïveté, trouvaient que le Saint Père ressemblait à Notre-Seigneur ; des protestants attendris disaient : "Voilà le plus grand homme de son siècle". Telle est la grandeur de la véritable société chrétienne, où Dieu se mêle sans cesse avec les hommes; telle est sur la force du glaive et du sceptre la supériorité de la puissance du faible, soutenu de la religion et du malheur (MDT, 2004 : I, 1049).

La nouveauté de cette activité de communion que le narrateur veut instaurer entre le lecteur et sa propre personne consiste en une promotion des thèmes bas appartenant à la culture populaire. Paradoxalement, la piété, l'aveuglement religieux, la naïveté et les superstitions populaires, jusqu'alors rabaissés en tant que preuves d'obscurantisme, peuvent situer le lecteur dans l'épuration et l'approfondissement de sa foi. Bien que la conquête de l'esprit critique ne passe pas uniquement par le martèlement dogmatique, elle n'apparaît pas non plus sous le signe de l'improvisation. Il est vrai que le redressement de la religion par la promotion des sentiments et de l'émotivité des fidèles est un facteur d'originalité et affermit la position de Chateaubriand en tant que précurseur du romantisme. Dans cette perspective, les émotions s'apparentent à l'expression de l'authenticité, de l'innocence et de la sincérité que le narrateur valorise et qu'il veut implanter dans la conscience du lecteur par la mise en scène de l'exemplarité d'une culture populaire. Toutefois, l'écrivain voit que la remémoration d'émotions religieuses doit se placer à la hauteur de la représentation vitale et visuelle. Cet enjeu revendique un traitement rhétorique sérieux pour que l'image du passé se réactualise devant les yeux du lecteur. En conséquence, la visualisation tend vers les résonances picturales de l'hypotypose et son cortège de figures rhétoriques qui, par les processus de contraste, de symétrie, de correction et d'ennoblissement permettent de dépeindre la beauté évangélique de la scène. Cependant, on note que l'émotivité atteint son paroxysme avec le procédé de la sermocination ${ }^{3}$. Cette figure ancienne semble transposer le lecteur dans un cadre

${ }^{3}$ La sermocination et l'hypotypose sont ainsi susceptibles de créer le même effet de présence et de participation imaginaire, possibilité qui s'offre au lecteur grâce à la position de l'instance 
imaginaire pour qu'il puisse participer à l'enthousiasme mystique des fidèles recevant la bénédiction papale: "Vive le pape ! Vive le chef de l'Eglise ! , «Dieu vous console !» et s'unissant dans les sentiments de révérence et d'attendrissement : "Voilà le plus grand homme de son siècle ». On en conclut que les procédés d'evidentia sont assignables à l'immédiateté de l'hallucination persuasive par laquelle le narrateur idéalise les vertus du peuple en frappant le lecteur par la beauté et la pureté de la religion chrétienne. D'ailleurs, les Mémoires abondent en images manifestant la supériorité du christianisme : la piété des premiers chrétiens, le faste, la richesse et l'exaltation de cérémonies religieuses, l'agenouillement solennel du peuple devant le Pape ont pour objectif de remplir le lecteur d'admiration et de le convaincre de la préexcellence et de la durabilité de la relion chrétienne.

\section{LE MEMORIALISTE ET LE SAVOIR EXISTENTIEL}

Dans le contexte de l'authenticité recherchée par les lecteurs des Memoires, il est primordial d'établir les éléments de la pensée chateaubrianesque qui s'assimilent à l'émanation du savoir. Selon la définition, le terme de " savoir » s'applique à " ce que l'on sait» et désigne l'« ensemble de connaissances » (Morvan et al., 1994 : 1016). Conformément à cette formulation, l'existence du savoir exclut toute possibilité d'hamlétisation et de spéculation dont l'absence est marquée dans l'œuvre par la récurrence du présent gnomique et de l'épiphrase : «En 1792, la fidélité au serment passait encore pour un devoir ; aujourd'hui, elle est devenue si rare qu'elle est regardée comme une vertu » (MDT, 2004: I, 466; c'est nous qui soulignons). Par ce subterfuge d'ordre rhétorique l'écrivain dépossède le lecteur de tout pouvoir herméneutique et procède à distribuer des connaissances sous forme de vérités générales :

J'ai eu l'honneur d'être dépouillé trois fois pour la légitimité : la première, pour avoir suivi les fils de saint Louis dans leur exil ; la seconde, pour avoir écrit en faveur des principes de la monarchie octroyée; la troisième, pour m'être tu sur une loi funeste au moment que je venais de faire triompher nos armes : la campagne d'Espagne avait rendu des soldats au drapeau blanc, et si j'avais été maintenu au pouvoir, j'aurais reporté nos frontières aux rives du Rhin. En me frappant on n'a frappé qu'un dévoué serviteur. L'ingratitude est à l'aise avec la fidélité ; cependant il peut y avoir tel homme moins soumis, telle circonstance dont il ne serait bon d'abuser; l'histoire le prouve (MDT, 2004 : II, 23-24; c'est nous qui soulignons).

narrative favorisant la poétique de l'image. Sur ces questions, voir également Grosperrin (2001 : 150-153). 
Effectivement, le narrateur chateaubrianesque jouit de la catégorisation de ses observations : par le passage de l'individualité à la généralité, son expérience personnelle se métamorphose en vérités atemporelles, objectives et absolues. Cependant, il est curieux de voir comment ces connaissances s'élèvent au rang du savoir, d'autant que la vision du monde de l'écrivain est rarement en conformité avec le point de vue du lecteur. Dans cette perspective, on revient sur le problème de la dynamique interractionnelle selon laquelle l'adhésion à la vision narrative passe par la capacité de susciter l'attention, en sorte que le lecteur s'intéresse à une cause comme si elle était la sienne ${ }^{4}$. Puisque la prise de l'intérêt par le public bénéficie généralement de connotations positives, elle offre l'opportunité de valoriser les contenus véhiculés par l'instance narrative. Ainsi, l'échelle des valeurs sur laquelle se situent dorénavant les connaissances transmises par l'écrivain s'oriente vers le partage du savoir. Ce rapport entre connaissance, valeur et savoir montre que le savoir ne saurait exister sans l'aptitude humaine à la croyance que connote explicitement la notion de «valeur ». Le savoir implique donc la nécessité de faire acquiescer le lecteur au système de valeurs du narrateur. Si l'univers de croyance du lecteur est en désaccord fondamental avec la vision du narrateur, celui-ci se voit obligé de recourir à la persuasion rhétorique afin d'inculquer ses idées au public. Nous constatons en effet l'obstination du narrateur chateaubrianesque à assimiler diverses formes de persuasion ${ }^{5}$. Du point de vue formel, la transmission du savoir s'exerce autant par la copia que par la brevitas, autant par la pratique de l'amplification des topoï que par l'amplification traditionnelle relevant de la fixité des figures rhétoriques telles que parallélisme, anaphore, énumération, accumulation, métaphore, comparaison etc. Grâce à ce double mouvement, les plus grandes idées, telles que la supériorité de la religion chrétienne, l'impératif de la probité politique, la prééminence du devoir patriotique ou la noblesse du passé, de ses rites et de son éducation sont revendiquées comme un savoir légitime à imposer. Nous remarquons que le narrateur se rend compte de la nécessité d'une appropriation de son discours pour qu'il puisse gagner l'adhésion de son public. Cependant, le figement argumentatif et l'absolutisation de la vérité auctoriale ne peuvent pas se confondre avec la transmission du savoir s'ils résident uniquement dans la mobilisation du dispositif formel. Or, selon la définition, le savoir cherche moins la forme que la connaissance en elle-même. En

\footnotetext{
${ }^{4}$ Chateaubriand ne cesse de rappeler au lecteur le principe de tua res agitur en rattachant ses exemples à l'universalité anthropologique de certaines valeurs qui caractérisent toute communauté, toute société, malgré le décalage temporel ou culturel. L'écrivain est convaincu entre autres que tout homme possède la faculté innée de discerner le bien et le mal.

${ }^{5}$ La richesse et la diversité des moyens rhétoriques renforçant l'idée de la supériorité morale du narrateur ne nous permettent pas d'aborder ici cette problématique dans toute son envergure. Nous nous limitons à signaler les stratégies les plus représentatives et récurrentes.
} 
conséquence, il est nécessaire que le narrateur passe par l'étape de la transmission du savoir s'il veut que son acquisition s'avère fiable aux yeux du public. On assite donc à un changement important qui affecte la notion du savoir au sein du genre des mémoires. Le savoir ne réside plus dans des connaissances raisonnables telles que le transmet le mouvement encyclopédiste ou la tradition scolaire solidement ancrée. Cependant, il serait risqué d'affirmer que les Mémoires représentent le cas isolé d'une telle dégradation. Matthieu Liouville indique que le même phénomène s'observe déjà au sein de la poésie romantique qui fait ruiner la notion classique de la sagesse (Liouville, 2009: 447). La lecture des Mémoires apporte une résonance nouvelle du savoir qui se distribue dorénavant à travers la personne du narrateur doté de crédibilité que lui assurent le vécu et l'acquis. Un tel détournement produit un effet avantageux sur le jugement porté sur l'activité scripturale de l'écrivain parce qu'il permet d'accorder à l'échec des attributs stables du succès : «Et moi qui me débats contre le temps, moi qui cherche à lui rendre compte de ce qu'il a vu [...], que suis-je entre les mains de ce Temps, de ce grand dévorateur des siècles que je croyais arrêtés, de ce temps qui me fait pirouetter dans les espaces avec lui ? » (MDT, 2004 : I, 1078-1079). En effet, même si Chateaubriand radicalise sa représentation littéraire en accentuant ses défaites cuisantes et la réalité ironique, cette remise en cause de sa compétence du mémorialiste a pour finalité de dissimuler des stratagèmes persuasifs $^{6}$. Le rapprochement continuel entre écrivain et lecteur s'effectue non seulement par la problématique universelle de dilemmes tels que la fuite du temps, le caractère inévitable de la vieillesse, la crainte de la mort, de l'oubli et du néant. La connivence se réalise notamment par la catégorisation des expériences personnelles lorsque le narrateur, grâce aux rudes leçons de sa propre existence, se transforme en détenteur de sagesse et peut initier le public inexpérimenté au savoir accumulé. Ainsi, le soulignement de sa propre faiblesse sert à la répartition d'un vaste savoir social et existentiel dont la légitimité dépasse le cadre systématique pour croître sur les expériences empiriques du narrateur.

Néanmoins, l'intersection entre savoir et expérience se complique lorsque la promotion du savoir comporte des implications morales qui interviennent au cœur des vérités sur la religion, le patriotisme, la responsabilité politique, historique, etc. Puisque l'appréhension du savoir du point de vue éthique est liée à l'obligation de se distinguer par les qualités morales, cette occurrence définit largement la responsabilité du discours prononcé par le narrateur ainsi que la nécessité de son honorabilité morale. Cet enjeu narratif se concrétise dans les

${ }^{6}$ De nombreux chercheurs se contentent de souligner la dispersion de la signification dans les Mémoires qui imiterait l'incapacité du narrateur à maîtriser les trames de son récit. Voir par exemple Roulin (2000 : 196). 
Mémoires par le maniement du dispositif rhétorique permettant l'ennoblissement de l'instance narrative aux yeux du public: «Le clergé, qui, j'ose le dire, me doit tant, ne m'aime point, ne m'a jamais défendu ni rendu aucun service. Mais qu'importe ? Il s'agit d'être juste et de voir ce qui convient à la religion et à la monarchie » (MDT, 2004 : II, 152). En somme, le narrateur chateaubrianesque balance adroitement entre l'exemplarité et le genus humile, ce qui lui permet, d'une part, de susciter continuellement l'émerveillement du public, de l'autre, de respecter le bon goût de la modestie ${ }^{7}$. Dans cet exemple, le savoir perce à travers la conviction que les devoirs patriotiques doivent dominer des animosités personnelles. La promotion du bien public revient inlassablement sous la plume de Chateaubriand et constitue la clef de voûte de son système de valeurs mis en scène par le retournement des procédés rhétoriques.

$\mathrm{Au}$ sein de cette virtuosité rhétorique, nous nous contentons de souligner deux stratégies narratives par lesquelles l'écrivain parvient à enchâsser le savoir dans la tangibilité de ses expériences. Premièrement, l'accroissement du savoir dans la conscience du lecteur est réalisable par la démonstration négative du discours et des actes déployés par le narrateur. En ce qui concerne l'emploi des figures rhétoriques, la démonstration négative s'appuie sur le contraste ou l'opposition sémantique. Cette juxtaposition antithétique déclenche des images frappantes et accusatrices lorsque la droiture morale du narrateur s'oppose explicitement à la corruption morale de ses contemporains : « Je préfère, dit-on, une famille à ma patrie : non, je préfère au parjure la fidélité à mes serments, le monde moral à la société matérielle; voilà tout» (MDT, 2004 : II, 638). Le dévoilement de la sagesse morale du narrateur est possible grâce à la binarité de l'antithèse : le blâme équivaut paradoxalement à la révélation implicite de son propre système de valeurs. Cependant, la pratique itérative de l'antithèse et des autres figures basées sur la tension sémantique, parmi lesquelles on repère le contraste, l'oxymore et le paradoxe, se réduit à l'insuffisance de la parole si le discours narratif ne se confirme pas dans l'authenticité de l'acte :

Tout le monde se retira des fenêtres ; j'y restai. Les assassins s'arrêtèrent devant moi, me tendirent les piques en chantant, en faisant des gambades, en sautant pour approcher de mon visage les pâles effigies. L'œil d'une de ces têtes, sorti de son orbite, descendait sur le visage obscur du mort; la pique traversait la bouche ouverte dont les dents mordaient le fer : "Brigands !" m'écriai-je, plein d'une indignation que je ne pus contenir, "est-ce comme cela que vous entendez la liberté ?” (MDT, 2004 : I, 291-292).

${ }^{7}$ Cicéron considère le genus humile comme l'équilibre entre le bon mot (facetice) et la raillerie (dicacitas). L'orateur doit garder cet équilibre dans le maniement des traits d'esprit (sales) de manière à : " éviter la bouffonerie et l'insolence, et d'une manière générale l'auteur doit respecter sa propre personne, l'auditoire et les circonstances ». Sur cette problématique, voir Bizer (2001: 19-29). 
Ainsi, le savoir n'est pas puisé uniquement dans la parole, mais également dans la noblesse du geste oratoire certifiant l'attitude inébranlable du narrateur à l'égard de la corruptibilité morale et ses effets vicieux. Le passage à l'action a des répercussions importantes sur la réactualisation du savoir prôné par le narrateur. Il prouve que le savoir dépasse le cadre purement théorique, esthétique ou persuasif et que la véritable exemplarité morale ne se plonge ni dans l'inertie, ni dans la résignation, mais trouve des moyens pour poursuivre sa lutte. Un autre point de ralliement qui s'instaure entre l'écrivain et le lecteur par la transmission du savoir moral nous renvoie à l'activité de l'indignatio ${ }^{8}$. À l'instar de l'antithèse, l'emportement de l'indignatio se caractérise par son rôle revendicateur dans la mesure où il permet le détachement explicite de l'immoralité ambiante. Grâce à la progression de l'indignatio, le narrateur chateaubrianesque peut extérioriser les obligations morales qui pèsent sur l'être humain. Selon sa vision, l'homme, conformément à l'universalité du savoir moral, est censé obéir à des valeurs éthiques telles que la dignité, l'honnêteté, le patriotisme, l'honneur, etc.

En second lieu, le narrateur réussit à stabiliser les impressions du lecteur par le recours à différentes formes de l'amplification qui englobe entre autres le martèlement morpho-syntaxique, le creusement sémantique et la thématisation des trames narratives par l'exploitation du lieu commun :

J'EUS d'abord l'idée de remettre au Roi le portefeuille des Affaires Etrangères, et de supplier Sa Majesté de le rendre au vertueux duc de Montmorency. Que de soucis je me serais épargnés! Que de divisions j'eusse épargnées à l'opinion ! L'amitié et le pouvoir n'auraient pas donné un triste exemple, et la légitimité serait peut-être encore là. Couronné de succès, je serais descendu du ministère de la manière la plus brillante, pour livrer au repos le reste de mes jours (MDT, $1948:$ III, 225) ${ }^{9}$.

Pourtant, le figement rhétorique ne se résout pas en persuasion rébarbative et vulgaire qui se cantonnerait dans la manipulation et la fausseté de la perspective. L'appropriation du dispositif rhétorique apparaît plutôt comme la tentative d'une adaptation du savoir à la postérité. Malgré la revendication du caractère universel que l'humanité voudrait concéder au savoir, l'écrivain est confronté à des obstacles naturels dans sa transmission lorsque le savoir se voit abaissé au rang d'un héritage inintelligible et tombé en désuétude. Chateaubriand est d'ailleurs très sensible à l'engloutissement de la condition humaine et de ses

\footnotetext{
${ }^{8}$ Selon Aristote, l'affliction devant l'injustice d'une punition ou l'illégitimité d'un succès qu'exprime l'orateur par le sentiment d'indignatio se distingue par une forte implication morale de cette action : "Tout événement qui ne répond pas au mérite est inique à nos yeux; et c'est là ce qui fait que nous attribuons même aux Dieux le sentiment de l'indignation ». Voir Aristote (1870 : 246-247).

${ }^{9}$ La censure exercée par l'entourage de l'écrivain et la suppression des extraits importants nous incite à nous référer ici à l'édition de Maurice Levaillant.
} 
artefacts dans le néant et l'oubli. Ainsi, l'activité rhétorique de l'écrivain dans les Mémoires, tout comme son engagement idéologique, s'inscrivent dans la recherche des universaux tantôt dans l'idée, tantôt dans la forme même. Du point de vue idéologique, l'écrivain tente de placer le savoir sous l'emprise des valeurs dominantes qu'il perçoit avant tout dans la continuité de la moralité. Quoiqu'il en soit, cet héritage reste difficile à manier vu le caractère incertain des présuppositions narratives concernant l'univers de croyance du lecteur. Du point de vue formel, ce péril de l'étrangeté doit être récompensé par la réduction de toute divergence par les arcanes de la persuasion, où l'intronisation du savoir s'instaure par la cohérence discursive de la vision du narrateur. Ainsi, le savoir moral subit le procédé de rhétorisation repérable notamment au niveau du foisonnement des lieux communs qui deviennent «un terrain d'entente stratégiquement choisi $»^{10}$. Le lieu commun, basé sur la probabilité et la notoriété du savoir à transmettre, est capable de mobiliser des cadres généraux de pensée, de conduite et de raisonnement, en sorte que les clichés d'un bon citoyen, d'un homme politique responsable ou d'un devoir communautaire s'inculquent dans la conscience du lecteur et s'érigent en modèle de son propre comportement au sein de la société. Par contre, l'accumulation du savoir par le narrateur devient la marque de la sagesse par laquelle il acquiert le droit de guider son public, de l'admonester et de le ramener dans le droit chemin.

Il est vrai que cet enchevêtrement de plans idéologique et formel permet d'associer la transmission du savoir à la capacité d'exercer un pouvoir sur le lecteur, pouvoir grâce auquel le narrateur récupère la permanence de sa voix auprès du public. En même temps, les circonstances ne sont pas indifférentes à la réception de l'œuvre littéraire et du savoir qu'elle véhicule. Paradoxalement, l'épanouissement du savoir participe pleinement de l'ennui de la condition humaine et de l'ébranlement continuel de ses systèmes. L'apeurement amène tout être humain à la recherche d'un remède à ses souffrances et Chateaubriand se rend parfaitement compte de cette symétrie ironique entre les générations successives. Il ne cesse de démontrer que l'Histoire entraîne les hommes dans sa course et les confronte à la cruauté d'expériences incommunicables et inimaginables. Tel est l'exemple du narrateur qui assiste au démantèlement de son univers qui n'est qu'un miroir brisé reflétant la chute vertigineuse de la foi, de la morale, de la décence et de tout ordre: "Nous n'avons pas une idée aujourd'hui de l'impression que les excès de la Révolution avaient fait sur les esprits en Europe, et principalement parmi les hommes absents de la France pendant la Terreur ; il me semblait, à la lettre, que j'allais descendre aux enfers » (MDT, 2004: I, 615). Cependant, l'écrivain est loin de réclamer le caractère

${ }^{10}$ Les techniques argumentatives utilisées par le lieu commun enjoignent le lecteur d'accepter l'inviolabilité des normes et des vérités générales valorisées par l'instance narrative. Voir sur ces questions Kibédi-Varga (1989: 49-50). 
exceptionnel de la Révolution. Au contraire, il reconvertit ses Mémoires en un véritable carrefour d'événements, d'expériences et de réflexions universels, prouvant que tout bouleversement historique émerge comme un processus inévitable, continu et itératif : "On ne cesse, disais-je, de s'étonner des événements ; toujours on se figure atteindre le dernier; toujours la révolution recommence » (MDT, 2004 : II, 639). Le caractère tragique et irrépressible de changements historiques préoccupe l'écrivain dans la mesure où le passage de la sacralisation au sacrilège altère la transmission des identités et du savoir. Chaque époque a ses effrois ; ainsi le lecteur se tourne d'autant plus vers l'écrivain qu'il ne se conforte plus dans ses propres certitudes. La confiance en l'expérience personnelle du narrateur est à l'origine d'une nouvelle entente. Cette fois-ci le savoir nécessite de convoquer la voix savante et expérimentée du mémorialiste. Le narrateur chateaubrianesque refuse cependant de s'allier aux aberrations morales de son époque et à la modélisation du savoir selon les principes de la société déformée. Au contraire, il s'attache à la réactualisation du savoir conformément aux valeurs traditionnelles qu'il affermit dans la conscience du lecteur par les différents procédés stylistiques basés sur la répétition et la récurrence. Puisque la répétition constitue une modalité essentielle de compréhension et d'apprentissage, son activité persuasive dans les Mémoires est soumise à la légitimisation du savoir provenant d'expériences existentielles de l'écrivain. Même s'il se trouve dans l'incapacité de dominer son passé, il détient pourtant le pouvoir d'influencer l'avenir en indiquant au lecteur le chemin de la dignité, du courage et du sacrifice qu'il promeut au détriment de l'inertie, de la médiocrité et de l'égoïsme.

\section{CONCLUSION}

La force des Mémoires réside dans la transmission de connaissances durables et universelles, ancrées dans les choix éthiques et la capacité de discernement entre le bien et le mal. D'une part, le narrateur chateaubrianesque jouit de la symétrie des préoccupations humaines en transformant sa personne et ses expériences tragiques en une source du savoir inestimable qu'il communique à un public égaré. De l'autre, il fonde l'universalité de son œuvre monumentale sur la supériorité de la religion chrétienne et de ses valeurs morales. Dans les Mémoires, l'interdépendance entre la littérature, le savoir et la religion s'inscrit dans la perception de la représentation littéraire en tant que promotion et prolongement des valeurs traditionnelles telles que famille, patrie, vie, mérite, ordre, autorité etc. Depuis des siècles, on participe à leur apparent démantèlement, et depuis des siècles le public se met en quête de la spiritualité, du réconfort et d'indicateurs moraux. Nonobstant cette convergence quasi naturelle, le narrateur s'assure la connivence avec son public par le recours à la persuasion rhétorique dont la force vertigineuse lui permet d' « imposer » sa vision du monde à la conscience du lecteur. 


\section{BIBLIOGRAPHIE}

ARISTOTE (1870). Rhétorique d'Aristote. Paris : Librairie philosophique de Ladrange.

BIZER, MARC (2001). Les lettres romaines de Du Bellay. Montréal : Les Presses Universitaires de Montréal.

Chateaubriand, François-Rene (1948). Mémoires d'outre-tombe. Édition critique par M. Levaillant. Paris : Édition du Centenaire.

- (2004). Mémoires d'outre-tombe. Édition critique par J.-C. Berchet. Paris : La Pochothèque.

GrosPERRIN, JEAN-PhILIPPE (2001). Accourez à ce spectacle de la foi : Économie de la scène dans la prédication classique. In M.-T. Mathet (éd.), La Scène : Littérature et Arts Visuels (pp. 149168). Paris : L'Harmattan.

Kibedi-VARGA, ARON (1989). Discours, récit, image. Liège - Bruxelles : Éditions Mardaga.

Liouville, Matthieu (2009). Les rires de la poésie romantique. Paris : Champion.

MOUKETE, FerdinAND FERrys (2012). La satire et sa rhétorique dans les Mémoires d'outretombe. Paris : Edilivre Collection Tremplin.

MORVAN, DANIELE et al. (1994). Le Robert pour tous. Paris : Le Robert.

QUINTILIEN (1989). De l'institution oratoire. Paris : Les Belles Lettres.

Roulin, JeAn-Marie (2000). La Clausule dans les Mémoires d'outre-tombe. In J.-C. Berchet, P. Berthier (eds.), Chateaubriand Mémorialiste, Colloque du cent cinquantenaire 1848-1998 (pp. 187-204). Genève : Droz.

Verlet, AgNes (2001). Les vanités de Chateaubriand. Paris : Droz. 
